\title{
ROMP Crosslinkers for the Preparation of Aliphatic Aerogels
}

Jeremy M. Lenhardt ${ }^{*}$, , Sung Ho Kima, Marcus A. Worsleya, Roald N. Leifa, Patrick G. Campbella, Theodore F. Baumanna, Joseph H. Satcher, Jr. a, b

aMaterials Sciences Division, Lawrence Livermore National Laboratory, 7000 East Avenue, Livermore, Callifornia 94550, United States

${ }^{b}$ Nanoscale Synthesis and Characterization Laboratory, Lawrence Livermore National

Laboratory, 7000 East Avenue, Livermore, Callifornia 94550, United States

* Corresponding author. E-mail address: lenhardt2@llnl.gov

\begin{abstract}
Ring opening metathesis polymerization (ROMP) using Grubbs second generation catalyst generates aliphatic gels from norbornene based crosslinkable monomers with a functionality of 2 or 3 . Supercritical drying of these gels $\left(\mathrm{CO}_{2}\right)$ leads to the formation of aerogels with densities as low as $4 \mathrm{mg} \mathrm{mL}^{-1}$. The microstructure was analyzed by SEM, and pore analyses by nitrogen adsorption. The use of crosslinkable monomers allows the straightforward synthesis of aerogels consisting of only carbon and hydrogen with $\mathrm{C}: \mathrm{H}$ ratios up to ca. $\mathrm{CH}_{1.58}$ with a finer microstructure than that of DCPD.
\end{abstract}

\section{Introduction}

Aerogels are an intriguing class of materials having such properties as large surface area [1], low density [2] and low thermal conductivity [3]. Interest in these materials has been based on their uses in thermal/acoustic insulation [4, 5], capacitors [1], and chemical/energy storage [6]. While many aerogels are synthesized from inorganic precursors to form materials with, for example, silica [3], transition metals [2, 7-9] or lanthanides [10], the development of organic aerogels is a newer endeavor. These include polyurethane [11], polyimide [12], polydicyclopentadiene [13], resorcinol-formaldehyde (RF) [14], cellulose [15] and polysaccharides [16]. Of interest to our group is the formation of high $\mathrm{CH}$ balance aerogels; namely aerogels consisting of only carbon and hydrogen with an atom ratio $>\mathrm{CH}_{1.3}$ and preferably $\mathrm{CH}_{2}$ with densities below ca. $40 \mathrm{mg} \mathrm{mL}^{-1}$. 
A review of the literature concerning CH-based aerogels highlights styrene/divinylbenzene [17], RF [14], graphene [18], dicyclopentadiene (DCPD) [13, $19,20]$ and poly(4-methylpentene) [21]. Notably, poly(4-methylpentene) retains a $\mathrm{CH}$ balance of $\mathrm{CH}_{2.0}$, though the final aerogel densities are high ( ca. $200 \mathrm{mg} \mathrm{mL}^{-1}$ ) and the microporous volume is less than ca. $3 \%$ of the total pore volume [21]. We previously reported [22] that hydrogenation of polydicyclopentadiene ( $p$ DCPD) gels and supercritical drying to aerogels gives low density aerogels (40-50 mg mL $\mathrm{m}^{-1}$ ) of an overall $>\mathrm{CH}_{1.5}$ atom balance with high surface areas (ca. $200 \mathrm{~m}^{2} \mathrm{~g}^{-1}$ ) and ca. 23 $\mathrm{nm}$ average pore diameters (though the distribution is quite broad). Since $p$ DCPD gels are easily obtained, and give 'low' density, small pore size aerogels, we prepared additional ring opening metathesis polymerization (ROMP) monomers of multiple crosslink functionality $(\mathrm{f}=2,3)$ and higher $(\mathrm{CH}>1.2$; vs. DCPD) initial $\mathrm{CH}$ balances.
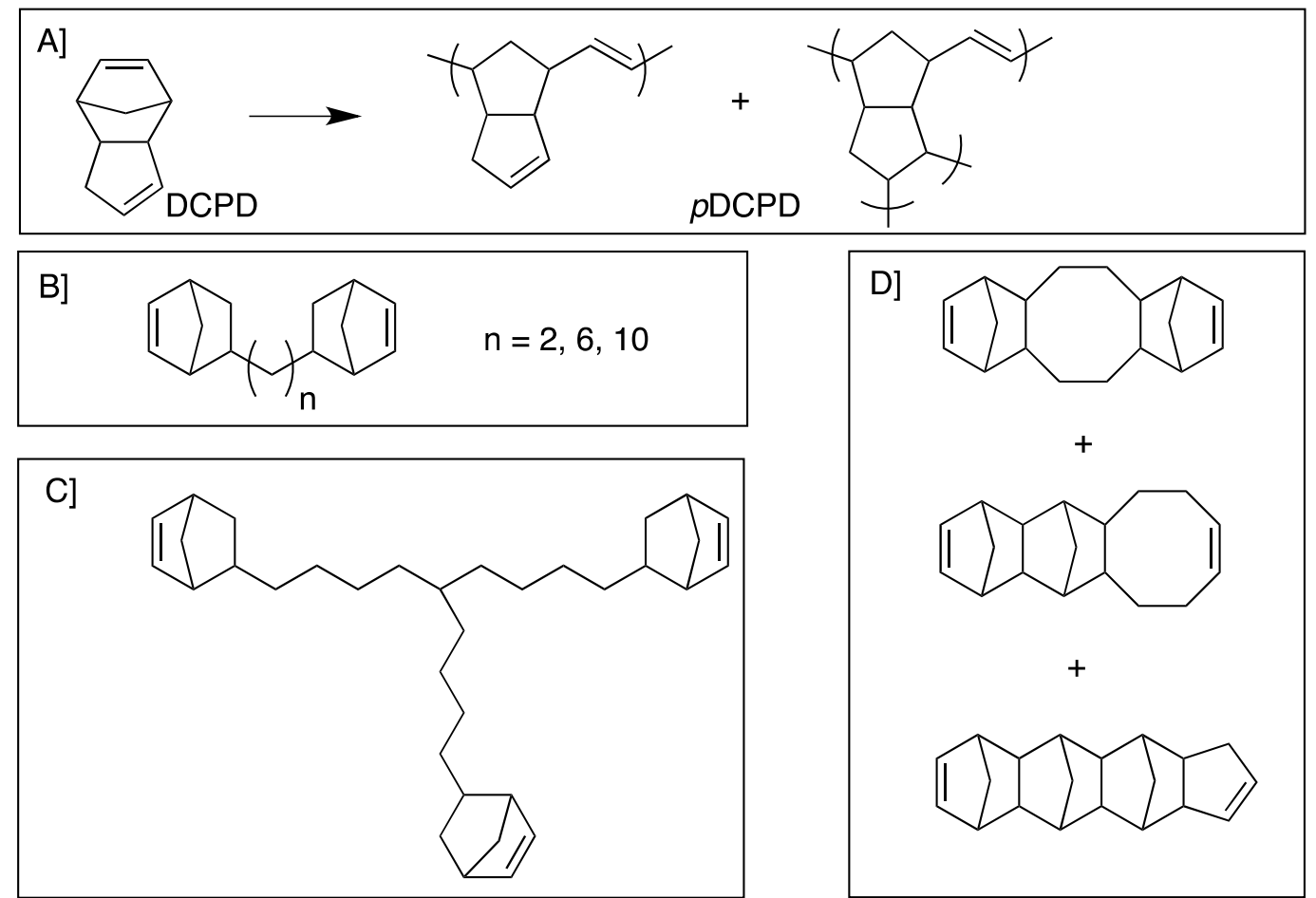

Figure 1. Monomers synthesized in this study are based on the ring opening metathesis polymerization (ROM) reaciton of DCPD [A]. Monomers synthesized for the current study include difunctional crosslinkers [B: $\mathrm{NB}_{2} \mathrm{NB}, \mathrm{NB}_{6} \mathrm{NB}, \mathrm{NB}_{10} \mathrm{NB}$, trifuctional crosslinker [C: $\mathrm{NB}_{3}$ ], and a monomer mixture [D: $\mathrm{NON}$ ] derived from the Diels-Alder reaction of cyclopentadiene with 1,5-cyclooctadiene.

The polymerization of DCPD gives mostly linear polymers [23] with a few interspersed crosslinks [24] that gives the gel the ability form a 3D matrix. The 
propensity of the ROMP reaction of DCPD to give linear polymer therefore limits the density of the DPCD gel; in our case at ca. $25-30 \mathrm{mg} \mathrm{mL}^{-1}$, and the density of the subsequent aerogel. Therefore, we looked to introduce norbornene monomers tethered between aliphatic linkers (Figure 1) that would serve as crosslinkers during ROMP polymerization by taking advantage of (a) cross-coupling reactions of primary [25] and secondary [26] Grignard reagents with primary alkyl bromides, and (b) Diels-Alder adducts from the reaction of cyclopentadiene with 1,5cyclooctadiene [27]. In the first, well-defined crosslinkable monomers are obtained while that of (b) leads to monomer mixtures that we were unable to separate and were used as prepared (Figure 1).

\section{Materials and methods}

\subsection{Materials}

Reagents were purchased from Sigma-Aldrich and used without purification unless otherwise noted (see SI). The preparation of Grignard reagents and all cross coupling chemistry was performed under nitrogen with dry solvents (anhydrous, inhibitor free grade, used as received) and reagents using standard Schlenck techniques.

\subsection{Sample preparation}

Ann apropriate amount of monomer was dissolved in toluene to reach the appropriate concentration, and 0.375 weight $\%$ Grubbs second generation catalyst was added to the mixture. The ring opening metathesis polymerization (ROMP) reaction was allowed to proceed for ca. 12-16 hours resulting in formation of a gel. After gelation, the gels were layered with toluene and allowed to age/react an additional 3 days. The gels were next placed into a large container of acetone with stirring, and solvent exchange into acetone was conducted for 4 days. Next, acetone exchanged gels were placed into a SPI-DRY critical point drier and charged with 900 psi liquid $\mathrm{CO}_{2}$ at ca. $12{ }^{\circ} \mathrm{C}$. Here, solvent exchange with liquid $\mathrm{CO}_{2}$ was allowed for 3 days with daily leaking of exchanged acetone from the critical point drier. After 3 days, the temperature and pressure of the $\mathrm{CO}_{2}$ liquid was raised above its' critical point to $40{ }^{\circ} \mathrm{C}$ and 1350 pis, respectively and held for 4 hours at these values to obtain the supercritical fluid-containing gels. After 4 hours the pressure was slowly released from the critical point drier (over ca. 2 hours) to obtain the final aerogels.

\subsection{Characterization}


SEM images were collected on a Jeol JSM-7401F scanning electron microscope at an acceleration voltage of $2 \mathrm{kV}$ in a lower secondary electron image (LEI) mode. Surface area, pore volume and pore diameter analyses were performed by Brunauer-Emmett-Teller (BET) and Barrett-Joyner-Halenda (BJH) methods using an ASAP 2000 surface area analyzer (Micromeritics Instrument Corporation). Samples were degassed for at least 16 hours at room temperature prior to analysis. Nitrogen adsorption data were then taken at five relative pressures from 0.05 to 0.20 at $77 \mathrm{~K}$ to calculate the surface area by BET theory. For BJH analyzes, average pore size and pore volume were calculated using data points from the desorption branch of the isotherm.

\section{Results and discussion}

3a. $N B_{X} N B$

$\mathrm{NB}_{\mathrm{x}} \mathrm{NB}$ gelation in toluene coincided with the alkyl tether length between reactive norbornene crosslinkers; $\mathrm{NB}_{2} \mathrm{NB}$ gelation was observed for 4 - $50 \mathrm{mg} \mathrm{mL}^{-1}$ solutions, 10 - $50 \mathrm{mg} \mathrm{mL}^{-1}$ for $\mathrm{NB}_{6} \mathrm{NB}$ (Figure 2) and $20-50 \mathrm{mg} \mathrm{mL}^{-1}$ for $\mathrm{NB}_{10} \mathrm{NB}$. After supercritical drying, the aerogels were carefully removed from their glass molds and the densities were directly measured. The lowest density aerogels were obtained from $\mathrm{NB}_{2} \mathrm{NB}\left(6 \mathrm{mg} \mathrm{mL}^{-1}\right), 16 \mathrm{mg} \mathrm{mL}^{-1}$ for $\mathrm{NB}_{6} \mathrm{NB}$ and $25 \mathrm{mg} \mathrm{mL}^{-1}$ for $\mathrm{NB}_{10} \mathrm{NB}$ (Table 1). SEM images (Figure 3) showed a finer, particulate network when compared to DCDP aerogels [20], which have a typically fibrous network morphology. This morphology is similar to that of a previously synthesized bisnorbornene aerogel with a phenyl ring linking two norbornene units [20].

Table 1. List of wet gel concentration $\left(\mathrm{mg} \mathrm{mL}^{-1}\right)$ and final aerogel densities $\left(\mathrm{mg} \mathrm{mL}^{-}\right.$ $\left.{ }^{1}\right)$ of $\mathrm{NB}_{\mathrm{x}} \mathrm{NB}$ systems. Bold values show lowest density aerogels achieved in the series.

\begin{tabular}{|c|c|c|c|c|c|}
\hline \multicolumn{2}{|c|}{$\mathrm{NB}_{2} \mathrm{NB}$} & \multicolumn{2}{c|}{$\mathrm{NB}_{6} \mathrm{NB}$} & \multicolumn{2}{c|}{$\mathrm{NB}_{10} \mathrm{NB}$} \\
\hline Wet Gel & Aerogel & Wet Gel & Aerogel & Wet Gel & Aerogel \\
\hline 5 & $\mathbf{6 . 2}$ & $n a$ & $n a$ & $n a$ & $n a$ \\
\hline 10 & 11 & 10 & $\mathbf{1 6}$ & $n a$ & $n a$ \\
\hline 20 & 31 & 20 & 30 & 20 & $\mathbf{2 5}$ \\
\hline 30 & 40 & 30 & 68 & 30 & 56 \\
\hline 40 & 63 & 40 & 130 & 40 & 63 \\
\hline 50 & 99 & 50 & 150 & 50 & 160 \\
\hline
\end{tabular}




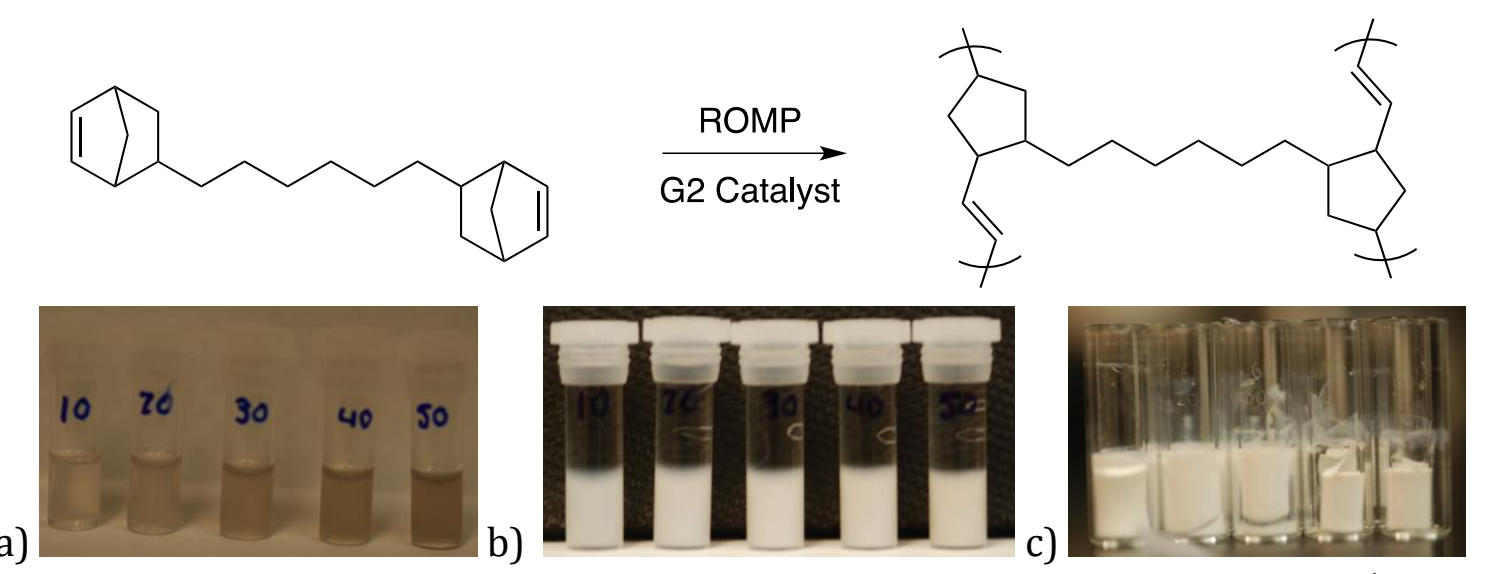

Figure 2. Preparation of $\mathrm{NB}_{6} \mathrm{NB}$ aerogels from solutions of $10-50 \mathrm{mg} \mathrm{mL}^{-1}$ (a) as prepared in toluene, (b) after solvent exchange into acetone and (c) supercritically dried aerogels. Wet gel concentrations $\left(\mathrm{mg} \mathrm{mL}^{-1}\right)$ are written on the vials.

a)
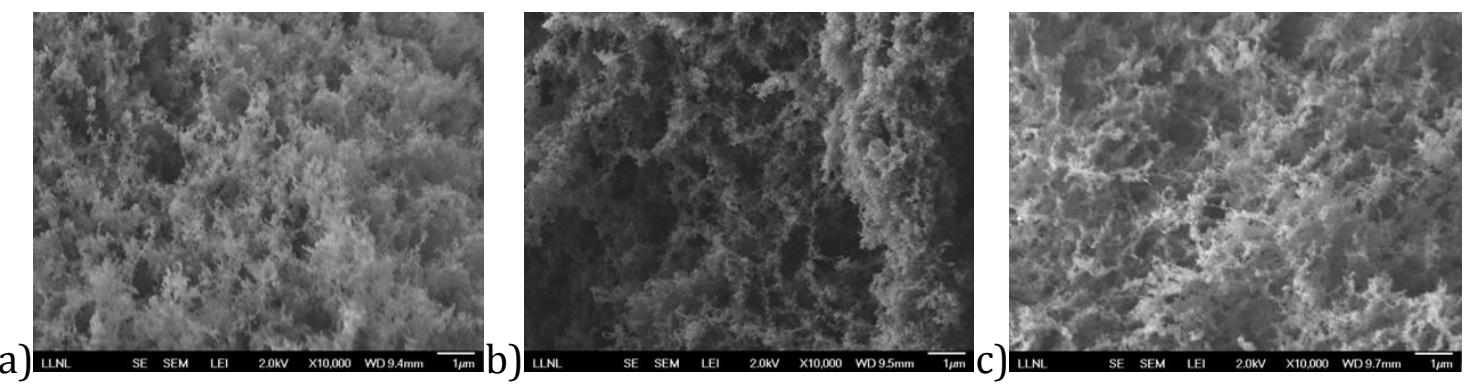

Figure 3. SEM images of (a) $\mathrm{NB}_{2} \mathrm{NB}$, (b) $\mathrm{NB}_{6} \mathrm{NB}$ and (d) $\mathrm{NB}_{10} \mathrm{NB}$.

Nitrogen adsorption analysis showed a decrease in surface area for crosslinkers of increasing alkyl tether length. For $30 \mathrm{mg} \mathrm{mL}^{-1}$ aerogels, these values decreased from 330 to 130 and $99 \mathrm{~m}^{2} \mathrm{~g}^{-1}$ for $\mathrm{NB}_{2} \mathrm{NB}, \mathrm{NB}_{6} \mathrm{NB}$ and $\mathrm{NB}_{10} \mathrm{NB}$ respectively. Pore volumes decreased in the same manner from $1.36,0.74$ and $0.69 \mathrm{~mL} \mathrm{~g}^{-1}$ while the average pore sizes were more similar at 54, 52 and $64 \mathrm{~nm}$.

3b. $N B_{3}$ and NON 

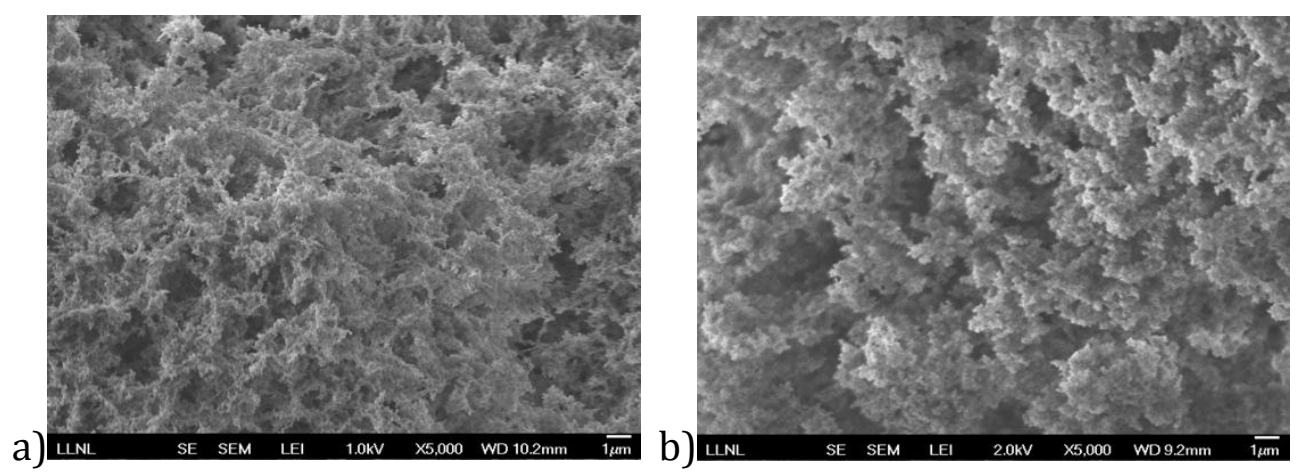

Figure 4. SEM images of $\mathrm{NB}_{3}$ (a) and NON (b) aerogels.

The reaction of a trifunctional $\mathrm{NB}_{3}$ monomer with Grubbs catalyst yielded low density aerogels down to $8.2 \mathrm{mg} \mathrm{mL}^{-1}$ from a $5 \mathrm{mg} \mathrm{mL} \mathrm{m}^{-1}$ wet gel precursor, and analysis of a $30 \mathrm{mg} \mathrm{mL}^{-1}$ aerogel showed a similar SEM microstructure to that of $\mathrm{NB}_{\mathrm{X}} \mathrm{NB}$ precursors (Figure 4). BET analysis showed this aerogel had similar values to that of $\mathrm{NB}_{\mathrm{X}} \mathrm{NB}$ systems as well; surface area of $200 \mathrm{~m}^{2} \mathrm{~g}^{-1}$, pore volume of $1.05 \mathrm{~mL}$ $\mathrm{g}^{-1}$ and an average pore size of $60 \mathrm{~nm}$. These values fall somewhat intermediate between that of $\mathrm{NB}_{2} \mathrm{NB}$ and $\mathrm{NB}_{6} \mathrm{NB}$ aerogels, indicating that the combination of crosslinker functionality ( $\mathrm{f}=2 \mathrm{vs} \mathrm{f}=3$ ) and alkyl tether length provide a measure for estimating both density and BET values. This relationship between monomer functionality and alkyl tether length led us to next consider the effect of molecular backbone 'rigidity' when considering/estimating aerogel formation. For example, alkyl tethers between NB units have larger degrees of rotational motion than, for example, cyclooctene or norbornene. The reaction of cyclopentadiene with 1,5cyclooctadiene provided a monomer mixture composed of alternating norbornene cyclooctene - norbornene (NBCONB), norbornene - bicyclo[2.2.1] heptane cyclooctene (NBHCO), and a dicyclopentadiene trimer $\left(\mathrm{D}_{3}\right)$ [27]. In this instance, we can only estimate the crosslinker functionality as between 1 and 2 . This monomer formed stable wet gets down to $4 \mathrm{mg} \mathrm{mL}^{-1}$, and after supercritical drying the dimensions of the aerogel within the mold remained unchanged and were difficult to remove from the glass vials at low concentrations. BET analysis of a $30 \mathrm{mg} \mathrm{mL}^{-1}$ NON aerogel showed the largest surface area in this NB-based series at $370 \mathrm{~m}^{2} \mathrm{~g}^{-1}$, pore volume of $0.93 \mathrm{~mL} \mathrm{~g}^{-1}$ and an average pore diameter of $50 \mathrm{~nm}$. Taken together, this series of NB-based monomers and their subsequent aerogel properties are shown in Figure 5. 

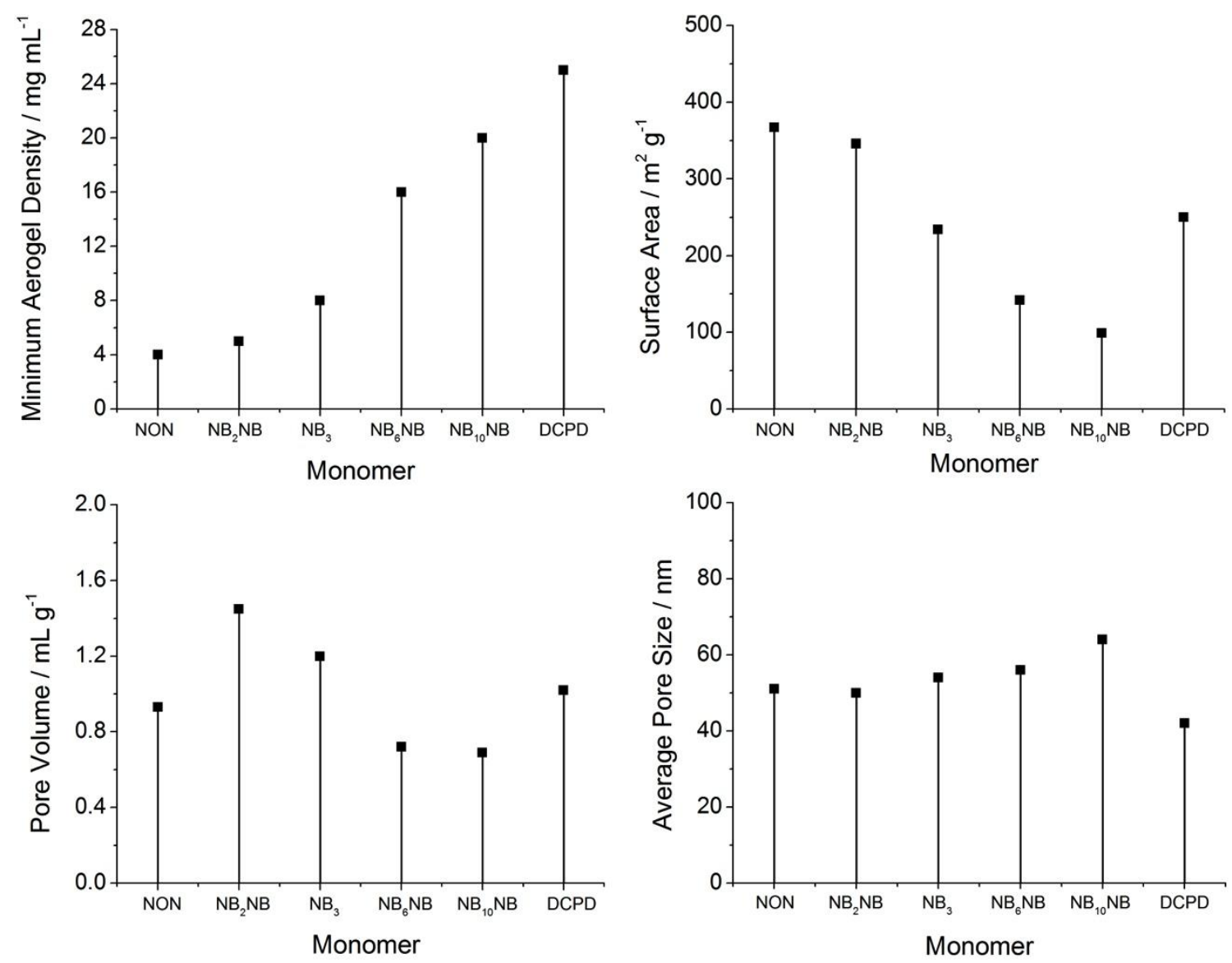

Figure 5. Graphs of the lowest achievable aerogel densities, surface areas, pore volumes and average pore sizes for monolithis in this study. DCPD aerogels included for reference.

\section{Conclusions}

The formation of aerogels of low density $\left(<10 \mathrm{mg} \mathrm{mL}^{-1}\right)$ are observed for ROMP monomers having a rigid polymer backbone (NON) and short aliphatic linker lengths between crosslinkable norbornene sites $\left(\mathrm{NB}_{2} \mathrm{NB}, \mathrm{NB}_{3}\right)$. An increase in linker length increases both the minimum wet gel density and final aerogel density. Surface areas are highest among the short aliphatic linker series, decreasing with increasing spacer length while pore volumes and the average pore sizes are similar. The monomers reported here are therefore versatile regents for the preparation of aliphatic aerogels.

\section{Acknowledgments}


This work was performed under the auspices of the U.S. Department of Energy by Lawrence Livermore National Laboratory under Contract DE-AC5207NA27344. IM release LLNL-JRNL-655922. 


\section{References}

[1] R. Saliger, U. Fischer, C. Herta, J. Fricke, High surface area carbon aerogels for supercapacitors, J. Non-Cryst. Solids, 225 (1998) 81-85.

[2] Y. Tang, K.L. Yeo, Y. Chen, L.W. Yap, W. Xiong, W. Cheng, Ultralow-density copper nanowire aerogel monoliths with tunable mechanical and electrical properties, Journal of Materials Chemistry A, 1 (2013) 6723-6726.

[3] S.S. Kistler, A.G. Caldwell, Thermal Conductivity of Silica Aërogel, Ind. Eng. Chem., 26 (1934) 658-662.

[4] B.E. Yoldas, M.J. Annen, J. Bostaph, Chemical Engineering of Aerogel Morphology Formed under Nonsupercritical Conditions for Thermal Insulation, Chem. Mater., 12 (2000) 2475-2484.

[5] M. Schmidt, F. Schwertfeger, Applications for silica aerogel products, J. Non-Cryst. Solids, 225 (1998) 364-368.

[6] H. Kabbour, T.F. Baumann, J.H. Satcher, A. Saulnier, C.C. Ahn, Toward New Candidates for Hydrogen Storage: High-Surface-Area Carbon Aerogels, Chem. Mater., 18 (2006) 6085-6087.

[7] A.E. Gash, T.M. Tillotson, J.H. Satcher, J.F. Poco, L.W. Hrubesh, R.L. Simpson, Use of Epoxides in the Sol-Gel Synthesis of Porous Iron(III) Oxide Monoliths from Fe(III) Salts, Chemistry of Materials, 13 (2001) 999-1007.

[8] A.E. Gash, J.H. Satcher Jr, R.L. Simpson, Monolithic nickel(II)-based aerogels using an organic epoxide: the importance of the counterion, J. Non-Cryst. Solids, 350 (2004) 145-151.

[9] B.C. Tappan, M.H. Huynh, M.A. Hiskey, D.E. Chavez, E.P. Luther, J.T. Mang, S.F. Son, Ultralow-Density Nanostructured Metal Foams: Combustion Synthesis, Morphology, and Composition, Journal of the American Chemical Society, 128 (2006) 6589-6594.

[10] T.M. Tillotson, W.E. Sunderland, I.M. Thomas, L.W. Hrubesh, Synthesis of lanthanide and lanthanide-silicate aerogels, J. Sol-Gel Sci. Technol., 1 (1994) 241-249.

[11] G. Biesmans, A. Mertens, L. Duffours, T. Woignier, J. Phalippou, Polyurethane based organic aerogels and their transformation into carbon aerogels, J. Non-Cryst. Solids, 225 (1998) 64-68.

[12] H. Guo, M.A.B. Meador, L. McCorkle, D.J. Quade, J. Guo, B. Hamilton, M. Cakmak, G. Sprowl, Polyimide Aerogels Cross-Linked through Amine Functionalized Polyoligomeric Silsesquioxane, ACS Applied Materials \& Interfaces, 3 (2011) 546-552.

[13] J. Lee, G. Gould, Polydicyclopentadiene based aerogel: a new insulation material, J. Sol-Gel Sci. Technol., 44 (2007) 29-40.

[14] R.W. Pekala, Organic aerogels from the polycondensation of resorcinol with formaldehyde, J. Mater. Sci., 24 (1989) 3221-3227.

[15] H. Jin, Y. Nishiyama, M. Wada, S. Kuga, Nanofibrillar cellulose aerogels, Colloids and Surfaces A.: Physiochem. Eng. Aspects, 240 (2004) 63-67.

[16] C.A. Garcia-Gonzalez, M. Alnaief, I. Smirnova, Polysaccharide-based aerogels - Promising biodegradable carriers for drug delivery systems, Carbohydrate Polymers, 86 (2011) 14.

[17] N.G. Borisenko, W. Nazarov, C.S.A. Musgrave, Y.A. Merkuliev, A.S. Orekhov, L.A. Borisenko, Characterization of divinyl benzene aerogels with density gradient using $X$-ray tomography technique, J Radioanal Nucl Chem, (2013) 1-4.

[18] M.A. Worsley, P.J. Pauzauskie, T.Y. Olson, J. Biener, J.H. Satcher, T.F. Baumann, Synthesis of Graphene Aerogel with High Electrical Conductivity, Journal of the American Chemical Society, 132 (2010) 14067-14069.

[19] C. Dawedeit, S.H. Kim, T. Braun, M.A. Worsley, S.A. Letts, K.J. Wu, C.C. Walton, A.A. Chernov, J.H. Satcher, A.V. Hamza, J. Biener, Tuning the rheological properties of sols for lowdensity aerogel coating applications, Soft Matter, 8 (2012) 3518-3521.

[20] S.H. Kim, M. Worsley, C. Valdez, S.J. Shin, C. Dawedeit, T. Braun, T. Baumann, S.A. Letts, S.O. Kucheyev, K.J. Wu, J. Biener, J. Satcher, A.V. Hamza, Exploration of the Versatility of Ring Opening Metathesis Polymerization: An Approach for Gaining Access to Low Density Polymeric Aerogels, RSC Advances, (2012).

[21] C. Daniel, J.G. Vitillo, G. Fasano, G. Guerra, Aerogels and Polymorphism of Isotactic Poly(4methyl-pentene-1), ACS Applied Materials \& Interfaces, 3 (2011) 969-977.

[22] J.M. Lenhardt, S.H. Kim, A.J. Nelson, P. Singhal, T.F. Baumann, J.H. Satcher Jr, Increasing the oxidative stability of poly(dicyclopentadiene) aerogels by hydrogenation, Polymer, 54 (2013) 542-547. 
[23] Y.-S. Yang, E. Lafontaine, B. Mortaigne, NMR characterisation of dicyclopentadiene resins and polydicyclopentadienes, J. Appl. Polym. Sci., 60 (1996) 2419-2435.

[24] T.A. Davidson, K.B. Wagener, D.B. Priddy, Polymerization of Dicyclopentadiene: A Tale of Two Mechanisms, Macromolecules, 29 (1996) 786-788.

[25] J. Terao, H. Todo, S.A. Begum, H. Kuniyasu, N. Kambe, Copper-Catalyzed Cross-Coupling Reaction of Grignard Reagents with Primary-Alkyl Halides: Remarkable Effect of 1-Phenylpropyne, Angewandte Chemie, 119 (2007) 2132-2135.

[26] C.-T. Yang, Z.-Q. Zhang, J. Liang, J.-H. Liu, X.-Y. Lu, H.-H. Chen, L. Liu, Copper-Catalyzed Cross-Coupling of Nonactivated Secondary Alkyl Halides and Tosylates with Secondary Alkyl Grignard Reagents, Journal of the American Chemical Society, 134 (2012) 11124-11127.

[27] S. Hara, Z.I.C.O.T.L. Endo, H.C.O.T.L. Mera, Methathesis polymerized copolymer, in, Google Patents, 1988. 